\title{
Total knee replacement with and without patellar resurfacing
}

\author{
A PROSPECTIVE, RANDOMISED TRIAL USING THE PROFIX TOTAL \\ KNEE SYSTEM
}

\author{
A. J. Smith, \\ D. J. Wood, \\ M.-G. Li
}

From the University of Western Australia, Nedlands, Australia
A. J. Smith, BSc, PhD, Research Fellow D. J. Wood, FRCS, FRACS, $M D$, Professor

M.-G. Li, MD, PhD, Senior Research Fellow School of Surgery and Pathology (Orthopaedics) University of Western Australia, Gate 3, Verdun Street, Nedlands, Western Australia 6009, Australia.

Correspondence should be sent to $\operatorname{Dr}$ A. J. Smith; e-mail: Anne.Smith@exchange.curtin. edu.au

(2008 British Editorial Society of Bone and Joint Surgery doi:10.1302/0301-620X.90B1. $18986 \$ 2.00$

$J$ Bone Joint Surg $[\mathrm{Br}]$ 2008;90-B:43-9. Received 4 December 2006; Accepted after revision 20 August 2007

We have examined the differences in clinical outcome of total knee replacement (TKR) with and without patellar resurfacing in a prospective, randomised study of 181 osteoarthritic knees in $\mathbf{1 4 2}$ patients using the Profix total knee system which has a femoral component with features considered to be anatomical and a domed patellar implant.

The procedures were carried out between February 1998 and November 2002. A total of 159 TKRs in 142 patients were available for review at a mean of four years ( 3 to 7$)$. The patients and the clinical evaluator were blinded in this prospective study. Evaluation was undertaken annually by an independent observer using the knee pain scale and the Knee Society clinical rating system. Specific evaluation of anterior knee pain, stair-climbing and rising from a seated to a standing position was also undertaken.

No benefit was shown of TKR with patellar resurfacing over that without resurfacing with respect to any of the measured outcomes. In 22 of 73 knees (30.1\%) with and 18 of 86 knees $(20.9 \%)$ without patellar resurfacing there was some degree of anterior knee pain $(p=0.183$ ). No revisions related to the patellofemoral joint were performed in either group. Only one TKR in each group underwent a re-operation related to the patellofemoral joint. A significant association between knee flexion contracture and anterior knee pain was observed in those knees with patellar resurfacing $(p=0.006)$.

While total knee replacement (TKR) is commonly carried out for end-stage degenerative arthritis of the knee, orthopaedic surgeons are still seeking clarification as to the indications for patellar resurfacing during this procedure. $^{1-4}$ Recent meta-analyses have been unable to relate the design of the arthroplasty to patellofemoral outcome. ${ }^{1,5,6}$ The design features of the femoral component may be an important consideration regarding the outcome after TKR when the patella has not been resurfaced. ${ }^{4,7,8}$ From the results of in vitro studies it appears that there may be optimal design factors for the femoral component, such as a deep-set, contoured femoral trochlea extending sufficiently proximally and posteriorly to maintain contact with the patella., ${ }^{9,10}$ However, in vitro studies after TKR do not exactly replicate the forces occurring at the joint in vivo, and cannot account for timedependent biological changes. Therefore, welldesigned clinical trials are necessary to confirm the clinical benefit of a more anatomicallydesigned patellofemoral joint in TKR.

We have examined the differences in the clinical outcome between TKR with and without patellar resurfacing using the Profix Total
Knee System (Smith \& Nephew Richards Inc., Memphis, Tennessee), which has a femoral component designed for compatibility with the native patella. It was thought that TKR with and without patellar resurfacing would result in comparable outcomes at a minimum followup of three years, given the anatomical design of the femoral component. The primary outcome compared was the difference in the knee pain score before and after surgery. Secondary outcomes were the incidence and severity of anterior knee pain after operation, the rate of re-operation, the Knee Society clinical rating system, ${ }^{11}$ the functional ability related to the patellofemoral joint and patient satisfaction.

Patients and Methods

Between February 1998 and November 2002, all patients with osteoarthritis (OA) undergoing primary TKR at two university-affiliated teaching hospitals were evaluated for inclusion in the study, which was approved by both the university and hospital ethics committees. Patients with inflammatory arthritis, a history of patellar fracture, patellectomy, patellofemoral instability or prior unicondylar knee 
Table I. Pre-operative clinical and operative status of patients undergoing total knee replacement with and without patellar resurfacing*

\begin{tabular}{|c|c|c|}
\hline & With resurfacing $(\mathrm{n}=87)$ & Without patellar resurfacing $(n=94)$ \\
\hline Mean age (yrs) (range) & $71.9 \quad(54.4$ to 88.1$)$ & $71.2 \quad(52.9$ to 84.9$)$ \\
\hline Gender (proportion of males) & 0.52 & 0.49 \\
\hline Side (proportion of right) & 0.49 & 0.49 \\
\hline Mean weight $(\mathrm{kg})$ (range) & $86.1 \quad(54.0$ to 125.0$)$ & $82.2 \quad(56.0$ to 125.0$)$ \\
\hline Mean height $(\mathrm{m})$ (range) & $1.67(1.48$ to 1.83$)$ & $1.65(1.42$ to 1.85$)$ \\
\hline Mean $\mathrm{BMI}^{\dagger}\left(\mathrm{kg} / \mathrm{m}^{2}\right)$ (range) & $31.0 \quad(21.5$ to 46.5$)$ & $30.0 \quad(20.5$ to 46.5$)$ \\
\hline Mean range of movement $\left({ }^{\circ}\right)$ & 7 to 112 & 6 to 111 \\
\hline Range of extension $\left({ }^{\circ}\right)$ & -2.0 to 23.0 & 0.0 to 15.0 \\
\hline Range of flexion $\left({ }^{\circ}\right)$ & 65.0 to 130.0 & 54.0 to 155.0 \\
\hline Pre-operative anterior knee pain present (number, \%) & $48(55)$ & $50(53)$ \\
\hline $\begin{array}{l}\text { Pre-operative anterior knee pain as predominant pain } \\
\text { (number, \%) }\end{array}$ & $31(36)$ & $33(35)$ \\
\hline \multicolumn{3}{|l|}{ Alignment $(\%)^{\ddagger}$} \\
\hline Varus $\left(<5^{\circ}\right)$ & $54(65)$ & $66(72)$ \\
\hline Normal $\left(5^{\circ}\right.$ to $\left.10^{\circ}\right)$ & 19 (23) & $16(17)$ \\
\hline Valgus $\left(>10^{\circ}\right)$ & $10(12)$ & $10(11)$ \\
\hline \multicolumn{3}{|l|}{ Approach (\%) } \\
\hline Mid-vastus & $42(48)$ & $41(44)$ \\
\hline Medial parapatellar & $43(50)$ & $47(50)$ \\
\hline Lateral & $2(2)$ & $6(6)$ \\
\hline \multicolumn{3}{|l|}{ Surgeon (\%) } \\
\hline 1 & $14(16)$ & $17(18)$ \\
\hline 2 & $13(15)$ & $14(15)$ \\
\hline 3 & $60(69)$ & $63(67)$ \\
\hline Lateral release (number, $\%)^{\ddagger}$ & $17(20)$ & $16 \quad(17)$ \\
\hline
\end{tabular}

replacement were excluded. A total of 164 patients with 181 TKRs were recruited and provided informed consent. The two treatment groups were similar with respect to clinical details and pre-operative clinical status (Table I).

The patients were randomised to undergo Profix TKR either with (87 knees) or without (94 knees) patellar resurfacing by the use of computer-generated random numbers. Those undergoing bilateral procedures were randomised to either right with and left without, or left with and right without patellar resurfacing. In no patient did both knees receive the same patellar treatment. The randomisation envelope was opened in the operating theatre.

Operative technique. Surgery was performed by one of three experienced surgeons (including DJW) or their trainees under supervision. All the components were cemented. A midline skin incision and a medial parapatellar, midvastus or lateral approach was used, with preservation of the infrapatellar fat pad. In the case of patellar retention a patelloplasty was performed, which involved only resection of marginal, protuberant osteophytes and loose flaps of cartilage. Patellar resurfacing was undertaken using a cemented, inset Profix-domed component. The height of the patella was measured before and after operation, and in no case differed by more than $2 \mathrm{~mm}$. Optimal patellar tracking was ensured by appropriate soft-tissue balancing, and a lateral release was performed at least $2.5 \mathrm{~cm}$ lateral to the lateral patellar border if the patella subluxed during passive testing of the range of movement. The TKRs with and without patellar resurfacing were comparable in terms of the operative variables of the surgical approach, the surgeon and lateral release at operation (Table I).

Clinical evaluation. Clinical evaluation was carried out before and after operation by one of two therapists (including AJS) who were blinded to the patient's randomisation. Evaluations were performed before and at 12 months after surgery, and then annually thereafter. The Knee Society clinical rating system, ${ }^{11}$ the knee pain scale ${ }^{12}$ and a knee diagram were used at each clinical assessment. The knee pain scale is a validated instrument for assessing both the severity and frequency of knee pain in OA. It consists of a five-point pain frequency subscale and a six-point pain intensity subscale applied to three transfer and three ambulation activities. The knee pain scale was chosen since it is potentially the most sensitive of available validated methods for evaluating any differences in the degree of anterior knee pain between the two groups. After operation, functional observations were made of the patients as they ascended and descended five steps of standard height and 
rose from a chair at knee height. Patient satisfaction was assessed by a validated satisfaction score described previously by Lingard et al, ${ }^{13}$ which included four questions related to relief from pain, functional ability and overall satisfaction.

A blinded, independent observer (MGL) measured the tilt, lateral displacement and thickness of the patella from skyline radiographs taken at $30^{\circ}$ of knee flexion, at the most recent follow-up, according to the method described by Gomes, Bechtold and Gustilo. ${ }^{14}$

Statistical analysis. This was performed using the SPSS version 12.0.1 statistical package (SPSS Inc., Chicago, Illinois). Categorical data were compared using the chi-squared test. Non-parametric statistics were used for analysis of continuous variables when data did not meet the assumptions of parametric tests. An independent Student $t$-test or the Mann-Whitney U test was used to analyse score differences between the two groups. Data from subjects with bilateral TKR were analysed using the Wilcoxon signed-rank test. Logistic regression analysis was used to assess associations between potential explanatory variables and the categorical, independent variable post-operative anterior knee pain. Residual analysis was performed and confirmed the validity of these models. For the main outcomes after operation the significance level was set at $\mathrm{p} \leq 0.05$.

Power analysis determined that a sample size of 180 gave a power of $92 \%$ to detect a ten-point difference in the mean change in the knee pain score between groups, which represented one half of an SD in change scores obtained from a pilot study of TKR patients. Since the study was one of equivalence, a confidence interval (CI) for the change score which fell within a point range of -10 to +10 was statistically significant evidence for equivalence.

\section{Results}

Clinical follow-up of three or more years was available in 159 knees (142 patients), 73 with patellar resurfacing and 86 without. The mean follow-up period was 4.37 years ( 3 to 7.03) and the mean length of follow-up was comparable for those knees with and without patellar resurfacing at 4.35 (3.0 to 7.03 ) and 4.37 (3.0 to 7.02) years, respectively. Seven patients, four with and three without resurfacing, withdrew from the study before the three-year follow-up because of ill health in four, moving away in two and problems with transport in one. Ten patients died before three years after operation for reasons unrelated to the surgery, seven with and three without patellar resurfacing. Two were lost to follow-up, one with and one without patellar resurfacing, before three years. These cases were excluded from the clinical follow-up analysis as were three knees revised for infection.

Revisions and re-operations. There were no revisions related to the patellofemoral joint in either group. In the group without patellar resurfacing, one arthroscopy was performed 30 months after operation for removal of a patellofemoral nodule. One arthroscopy was performed in a knee with patellar resurfacing six months after operation for investigation of a locked knee. No loose bodies or cement was found, but some adhesions were released, resulting in an improved range of movement.

The three infected TKRs underwent two-stage revision, two with patellar resurfacing at 34 and 40 months and one without at 26 months, respectively, after surgery.

In the group with patellar resurfacing, an arthrotomy was performed at five weeks and an arthroscopic washout at six weeks after operation, both for infection. These reoperations for infection were not the same as those carried out for infection described previously. There were three reoperations unrelated to the patellofemoral joint in the group without patellar resurfacing. These were an exchange to a conforming plus tibial insert at seven months because of instability, removal of a posterior ganglion at 15 months, and an arthroscopic washout and exchange of the tibial insert performed for infection at four months after operation.

Knee pain scale. Both treatment groups showed highly significant improvements in the score in the knee pain scale (paired samples $t$-test, $\mathrm{p}<0.001$ in both). The postoperative scores were highly skewed, with the median score (interquartile range (IQR)) being 100.0 points (37.0) for TKR with resurfacing and 100.0 points (23.6) for TKR without patellar resurfacing. Those with patellar resurfacing improved by a mean of 47.7 points (-7.4 to 92.6$)$ and those without patellar resurfacing by a mean of 48.7 points $(-3.7$ to 87.0$)$. The difference in the mean change score of 1.0 point between TKR with and without patellar resurfacing was not statistically significant (independent $t$-test, $\mathrm{p}=0.797,95 \%$ CI -6.6 to 8.5 ) (Table II).

Anterior knee pain. At the latest follow-up, 22 of 73 knees (30.1\%; 95\% CI 19.6 to 40.7$)$ with patellar resurfacing and 18 of $86(20.9 \%$; $95 \%$ CI 12.3 to 29.5$)$ without resurfacing had any degree of anterior knee pain identified from the knee diagram and knee pain scale and a score of 45 or less on the Knee Society knee score pain subscale. The difference in proportions was not significant $(-9.2 \%$; chisquared test, $\mathrm{p}=0.182,95 \% \mathrm{CI}-22.8$ to +4.4$)$. This corresponded to an OR of 0.61 (logistic regression, $\mathrm{p}=0.184$, $95 \%$ CI 0.30 to 1.26 ) for anterior knee pain in TKR without resurfacing as compared with TKR with patellar resurfacing.

Ten of $73(13.7 \%)$ knees with patellar resurfacing and five of $86(5.8 \%)$ without had moderate to severe anterior knee pain identified from the knee diagram and knee pain scale and a score of 20 or less on the Knee Society score pain subscale, which was not a statistically significant difference $(-7.9 \%$, chi-squared test, $\mathrm{p}=0.090 ; 95 \%$ CI -17.2 to 1.4). For those TKRs which were painful anteriorly, the mean knee pain scale score was 56.9 (29.6 to $94.4, \mathrm{n}=22$ ) and 61.5 (22.2 to $94.4, \mathrm{n}=18)$ for those with and without patellar resurfacing, respectively. This difference was not statistically significant (4.6; independent $t$-test, $\mathrm{p}=0.440$, $95 \%$ CI 7.4 to 16.6 ). Similarly, the mean Knee Society score 
Table II. Patient categories and pre-operative, post-operative, and pre- to post-operative change in clinical scores of all knees of patients undergoing total knee replacement with or without patellar resurfacing, with a minimum follow-up of three years

\begin{tabular}{|c|c|c|c|c|}
\hline & $\begin{array}{l}\text { Patellar resurfacing } \\
(n=73)\end{array}$ & $\begin{array}{l}\text { No patellar resurfacing } \\
(\mathrm{n}=86)\end{array}$ & $\begin{array}{l}\text { Difference and } 95 \% \mathrm{Cl}^{*} \\
\text { for difference }\end{array}$ & p-value ${ }^{\dagger}$ \\
\hline \multicolumn{5}{|l|}{ Category ${ }^{\ddagger}$ (number, \%) } \\
\hline$A$ & $27(37)$ & $34(39)$ & & \\
\hline B & $4(5)$ & $10(12)$ & & \\
\hline $\mathrm{C}$ & $42(58)$ & $42(49)$ & & \\
\hline \multicolumn{5}{|l|}{ Knee pain scale ${ }^{\S}$} \\
\hline Pre-operative (mean, SD) & $36.2(16.6)$ & $40.0(15.0)$ & & \\
\hline Post-operative (median, IQR & $100.0(37.0)$ & $100.0(23.6)$ & & \\
\hline Change (mean, SD) & $47.7(25.0)$ & $48.7(23.2)$ & $1.0(-6.6$ to +8.5$)$ & 0.797 \\
\hline \multicolumn{5}{|l|}{ Knee Society score ${ }^{\S}$} \\
\hline Pre-operative (mean, SD) & $39.7(18.9)$ & $39.0(13.8)$ & & \\
\hline Post-operative (median, IQR) & $92.0(12.0)$ & $93.0(11.0)$ & & \\
\hline Change (mean, SD) & $46.2(20.1)$ & $50.0(16.8)$ & $3.8(-2.1$ to +9.7$)$ & 0.202 \\
\hline \multicolumn{5}{|l|}{ Knee Society function score $e^{\S}$} \\
\hline Pre-operative (mean, SD) & $51.9(17.1)$ & $51.7(16.4)$ & & \\
\hline Post-operative (median, IQR) & $60.0(30.0)$ & $70.0(46.0)$ & & \\
\hline Change (mean, SD) & $14.4(19.3)$ & $18.6(19.5)$ & $4.1(-2.0$ to +10.3$)$ & 0.184 \\
\hline \multicolumn{5}{|c|}{$\begin{array}{l}\text { * } 95 \% \mathrm{Cl}, 95 \% \text { confidence interval } \\
\dagger \text { independent } t \text {-test } \\
\text { ‡A, unilateral or bilateral TKR; B, contralateral knee symptomatic; C, multi-articular arthritis or medical infirmity } \\
\S \text { maximum score } 100 \text { (best), minimum } 0 \text { (worst) } \\
\text { I IOR, interquartile range }\end{array}$} \\
\hline
\end{tabular}

Table III. Post-operative clinical scores (median, interquartile range (95\% confidence interval of median)) of 16 patients undergoing bilateral total knee replacement with and without patellar resurfacing

\begin{tabular}{|c|c|c|c|}
\hline & Patellar resurfacing & No patellar resurfacing & p-value* \\
\hline Knee Society score $^{\dagger}$ & $91.5,11.2$ (86.6 to 96.0 ) & $90.5,12.0$ (84.0 to 95.0$)$ & 0.160 \\
\hline Knee pain scale ${ }^{\dagger}$ & $100.0,9.7 \quad(93.7$ to 100.0$)$ & $100.0,19.4(83.5$ to 100.0$)$ & 0.249 \\
\hline Satisfaction score ${ }^{\dagger}$ & $100.0,15 \quad(90.3$ to 100.0$)$ & $100.0,0 \quad(100.0$ to 100.0$)$ & 0.655 \\
\hline
\end{tabular}

pain subscale scores were not significantly different for those with and without patellar resurfacing which were painful anteriorly, $28.2(10$ to $45, \mathrm{n}=22)$ and $35.6(10$ to $45, \mathrm{n}=18$ ) respectively; independent $t$-test, $\mathrm{p}=0.125,95 \%$ CI for difference -2.1 to 16.9 ).

Anterior knee pain was reported in five of the 16 patients who had undergone bilateral TKR with patellar resurfacing in one knee. Four of these had pain of the same intensity according to the Knee Society score pain subscale in both the TKR with and without patellar resurfacing. One patient reported mild anterior knee pain in the TKR without patellar resurfacing only. When those patients who had undergone bilateral TKR with patellar resurfacing in one knee only were analysed, no statistical differences were detected in the post-operative knee pain scale scores (Wilcoxon signed-rank test, $\mathrm{p}=0.249$; Table III).

Predictors of anterior knee pain. To identify predictive factors for post-operative anterior knee pain in each group, TKRs with and without patellar resurfacing were considered separately using a stepwise logistic regression model (backward likelihood ratio method ${ }^{15}$ ) after controlling for age and gender. Variables considered for inclusion were weight, height, the body mass index (BMI), lateral patellar release, flexion contracture and the presence of pre-operative anterior knee pain. In the case of TKR with patellar resurfacing, the presence of a flexion contracture was retained in the model after controlling for age and gender (logistic regression analysis, $p=0.006$, OR $9.73,95 \%$ CI 1.93 to 48.99$)$. None of the considered variables was predictive of post-operative anterior knee pain in the case of TKR without patellar resurfacing.

Knee Society clinical rating system. Both TKRs with and without patellar resurfacing showed highly significant improvement in the Knee Society scores (paired samples $t$-test, $\mathrm{p}<0.001$ in both groups). Post-operative scores were highly skewed with the median score (IQR) being 92.0 (12.0) points for TKR with patellar resurfacing and 93.0 
Table IV. Patellofemoral functional observations of patients undergoing total knee replacement with and without patellar resurfacing, by number and percentage

\begin{tabular}{|c|c|c|c|c|}
\hline Activity & Categories & $\begin{array}{l}\text { Patellar resurfacing* } \\
(n=72)\end{array}$ & $\begin{array}{l}\text { No patellar resurfacing } \\
(\mathrm{n}=\mathbf{8 6})\end{array}$ & p-valuet \\
\hline \multirow[t]{4}{*}{ Chair rising } & Able to rise with ease without use of arms & $41(56.9)$ & $54(62.8)$ & 0.737 \\
\hline & Able to rise with ease with use of arms & $24(33.4)$ & $24(27.9)$ & \\
\hline & Able to rise with difficulty & $7(9.7)$ & $8(9.3)^{\ddagger}$ & \\
\hline & Unable & $0(0.0)$ & $0(0.0)^{\ddagger}$ & \\
\hline \multirow[t]{3}{*}{ Stair ascent - rail } & No rail or rail for balance only & $41(56.9)$ & $57(66.2)$ & 0.229 \\
\hline & Rail for weight support & $31(43.1)$ & $28(32.6)$ & \\
\hline & Unable & $0(0.0)$ & $1(1.2)$ & \\
\hline \multirow[t]{2}{*}{ Stair ascent - lead leg§ } & Leads with operated leg or in reciprocal manner & $61(84.7)$ & $72(83.7)$ & 0.998 \\
\hline & Leads with non-operated leg & $11(15.3)$ & $13(15.1)$ & \\
\hline \multirow[t]{3}{*}{ Stair descent - rail } & No rail or rail for balance only & $36(50.0)$ & $52(60.5)$ & 0.187 \\
\hline & Rail for weight support & $36(50.0)$ & $33(38.3)$ & \\
\hline & Unable & $0(0.0)$ & $1(1.2)$ & \\
\hline \multirow[t]{2}{*}{ Stair descent - lead leg ${ }^{\S}$} & Leads with non-operated leg or in reciprocal manner & $50(69.4)$ & $66(76.7)$ & 0.244 \\
\hline & Leads with operated leg & $22(30.6)$ & $19(22.1)$ & \\
\hline
\end{tabular}

* data is missing for one knee

† chi-squared test

‡ merged for chi-squared analysis

$\S$ data is missing for one knee without patellar resurfacing

(11.0) points for TKR without patellar resurfacing. Those with patellar resurfacing improved by a mean of 46.2 points $(-3.0$ to 81.0$)$ and those without by a mean of 50.0 points $(-20.0$ to 83.0$)$. The difference in the mean change score of 3.8 points between TKR with and without patellar resurfacing was not statistically different (independent $t$-test, $\mathrm{p}=0.202,95 \%$ CI -2.1 to +9.7 ; Table II).

Additionally, there was no statistically significant difference between TKR with and without patellar resurfacing postoperatively in terms of the subscores of the Knee Society score (Mann-Whitney U test, $\mathrm{p}=0.098$ to 0.999 ). The 16 patients who had undergone bilateral TKR with patellar resurfacing in one knee only did not show a difference in the mean score between knees (Wilcoxon signed-rank test, $\mathrm{p}=0.160$ ) (Table III).

Both treatment groups had a highly significant improvement in Knee Society function scores (paired samples $t$-test, $\mathrm{p}$ $<0.001$ in both groups). After surgery the median score (IQR) was 60.0 points (30.0) for those with patellar resurfacing and 70.0 points $(46.0)$ for those without patellar resurfacing. Those with patellar resurfacing improved by a mean of 14.4 points (-35.0 to 55.0) and those without patellar resurfacing by a mean of 18.6 points (-20.0 to 55.0$)$. The difference in the mean change score of 4.1 points between TKR with and without patellar resurfacing was not statistically different (independent $t$-test, $\mathrm{p}=0.184,95 \% \mathrm{CI}-2.0$ to +10.3 ) (Table II).

Functional ability. With the numbers available, no statistically significant differences were observed between TKR with and without patellar resurfacing with regard to the performance on functional tasks (Table IV).
Satisfaction. Most patients obtained the maximum of 100 for the satisfaction score. A total of 19 of 71 (26.8\%; data missing for two knees) TKRs with patellar resurfacing and 15 of $86(17.4 \%)$ TKRs without patellar resurfacing, had a satisfaction score less than 100 . This did not represent a significant difference in proportions (chi-squared test, $\mathrm{p}=0.158)$. Of those knees with which patients were less than fully satisfied, the mean satisfaction score was 50.3 (8.0 to 92.0 ) in the group with and 62.9 (8.0 to 92.0$)$ in the group without patellar resurfacing. The mean difference of 7.9 was not significantly different (independent $t$-test, $\mathrm{p}=0.118$, $95 \%$ CI -3.4 to +28.6 ). The 16 patients who had undergone bilateral TKR with patellar resurfacing in one knee only did not show greater satisfaction with one knee over the other (Wilcoxon signed-rank test, $\mathrm{p}=0.655$ ) (Table III).

Radiological findings. In 65 of $73(89.0 \%)$ patients with patellar resurfacing and in 78 of $86(90.7 \%)$ of those without radiographs were available for analysis from the latest clinical follow-up. The mean patellar thickness $25.9 \mathrm{~mm}(95 \%$ CI 25.0 to 26.8 ) in those with and $24.3 \mathrm{~mm} \mathrm{(95 \%} \mathrm{CI} 23.5$ to 25.0 ) in those without patellar resurfacing. The mean difference of $1.6 \mathrm{~mm}$ was statistically significant (independent $t$ test, $\mathrm{p}=0.007,95 \%$ CI 0.5 to 2.8 ). The mean patellar tilt was also significantly greater in those knees with patellar resurfacing $\left(3.6^{\circ}, 95 \%\right.$ CI 2.7 to 4.4$)$, than in those without $\left(2.5^{\circ}, 95 \%\right.$ CI 1.8 to 3.1$)$ with a mean difference of $1.1^{\circ}$ (independent $t$-test, $\mathrm{p}=0.042,95 \%$ CI 1.01 to 2.2 ). However, no association between patellar thickness or patellar tilt and post-operative anterior knee pain was detected in knees with and without patellar resurfacing. 
Although knees without patellar resurfacing displayed higher mean lateral displacement $(3.9 \mathrm{~mm}, 95 \%$ CI 3.4 to 4.4) than those without $(3.1 \mathrm{~mm}, 95 \%$ CI 2.4 to 3.7$)$ the difference of $0.8 \mathrm{~mm}$ was not statistically significant (independent $t$-test, $\mathrm{p}=0.056,95 \% \mathrm{CI}$ of difference 0.0 to 1.6 ). However, in knees with patellar resurfacing, lateral displacement of more than $5 \mathrm{~mm}$ was significantly associated with post-operative anterior knee pain (logistic regression, $\mathrm{p}=$ 0.037, OR $4.20,95 \%$ CI 1.09 to 16.19 ) whereas in those knees without patellar resurfacing, no statistically significant association was detected (logistic regression, $\mathrm{p}=0.397$, OR $1.64,95 \%$ CI 0.52 to 5.18$)$.

\section{Discussion}

In this prospective, randomised study, analysis of the primary outcome measure of the knee pain scale showed no difference between TKR with and without patellar resurfacing in the Profix design. The 95\% CI for the difference in change of the knee pain scale score between TKR with and without patellar resurfacing was -6.6 to +8.5 points, which was within the interval of -10 to +10 points which we considered to be clinically reasonable to assume equivalence.

The OR was 0.61 (95\% CI 0.30 to 1.26$)$ for anterior knee pain in TKR without patellar resurfacing compared with that with resurfacing. Therefore, we cannot conclude that the risk of anterior knee pain is no greater without patellar resurfacing than with patellar resurfacing using the current design. However, the results are in contrast to a previous prospective, randomised study using the MillerGalante II design (Zimmer, Warsaw, Indiana), performed with the same length of follow-up. ${ }^{16}$ In that study, the incidence and severity of anterior knee pain were significantly greater in those TKRs without patellar resurfacing (39 of $127,30.7 \%$ ) than in those with patellar resurfacing (15 of 91, $16.5 \%)$, which corresponds to an OR of 2.24 (95\% CI 1.15 to 4.39 ). The current study had $72 \%$ power to detect an effect of this magnitude.

These contrasting results may be a result of differences in design between the two femoral components used. The femoral components of the Miller-Galante II and the Profix Total Knee System are of asymmetrical design with a proximally and anteriorly elevated lateral flange. However, the Miller-Galante II component has flat-shaped condyles, and consequently an angular trochlear groove, whereas the trochlear groove of the Profix blends more smoothly with its round-shaped condyles. Recent meta-analyses have concluded that patellar resurfacing is a superior technique in terms of the incidence of anterior knee pain., ${ }^{2,5,6,17}$ However, these reviews were unable to address the hypothesis that different effect sizes may be the result of differences in prosthetic design, because of lack of detailed information on individual patients. ${ }^{2,5,6}$

It must be emphasised that our study evaluates shortterm results only, and that it is possible that the results of TKR without patellar resurfacing will deteriorate with time. The primary aim of our study, however, was to eval- uate the early results using a prosthesis with a femoral component designed to be compatible with the native patella, and to compare these with the early results of a previous trial using a femoral component not providing an optimal surface for the native patella, performed at the same centre, with the same follow-up time. As such, our study adds weight to the argument that design influences the outcome of the patellofemoral joint after TKR.

The patellar component used with the Miller-Galante II system is an onset, 'hat-shaped' design with a raised central portion and a peripheral flat area. It articulates with the 'box-shaped' femoral trochlea. The patellar component used with the Profix system is an inset, dome-shaped design. The relative superiority of an oval dome design versus an anatomical design remains unclear. ${ }^{4}$ The relatively high rates of anterior knee pain in TKR with patellar resurfacing in the current study are similar to those reported in a recent longer term follow-up of a prospective, randomised trial of patellar resurfacing versus non-resurfacing using a similar anatomical femoral component with a domed patellar prosthesis. ${ }^{18}$ Laboratory-based studies have suggested that a domed patellar component articulating with an anatomical femoral component may be subjected to greater stresses in vivo than more conforming designs. ${ }^{19-23}$ However, since our study does not involve a direct comparison between designs of component, the evidence for poorer in vivo performance of the domed design remains inconclusive.

Flexion contracture was a risk factor for anterior knee pain in TKR with patellar resurfacing in our study $(p=0.006)$. Patellofemoral pain may ensue because of increased joint contact stress secondary to the increased joint reaction force. However, knee flexion contracture has not been identified as a factor associated with anterior knee pain in recently reported, randomised studies. ${ }^{16,24,25}$ There is the possibility with the use of a medialised, inset patellar component, that the lateral patellar facet may be left uncovered, resulting in its contact with the lateral flange of the femoral component, possibly leading to anterior knee pain. Since the position of the patellar component on the patellar surface was not measured in our study, this possible mechanism of anterior knee pain in TKR with patellar resurfacing cannot be verified. We noted that lateral patellar displacement was significantly associated with anterior knee pain in those TKRs with patellar resurfacing, despite this group having less lateral patellar displacement than those without patellar resurfacing. This raises the possibility that excessive lateral contact of the patella may be a factor in anterior knee pain in TKR with patellar resurfacing. However, two limitations of our study were the low power available to detect any association between patellar position and post-operative anterior knee pain and the fact that the position of the patellar component on the patella was not measured. It may be that the latter is important to the clinical outcome in TKR with a domed patellar component, despite the fact that, in theory, a domed component should 
be less susceptible to malposition because of its spherical shape.

Altered kinematics in the resurfaced patellofemoral joint may play a role in the genesis of anterior knee pain. A six-camera computerised gait analysis of a subset of the patients in our study revealed a trend toward decreased knee extension at heel-strike 0in those knees with patellar resurfacing $\left(10^{\circ}\right.$ vs $\left.7^{\circ}, \mathrm{p}=0.023\right) .{ }^{26}$ Furthermore, in vivo fluoroscopic studies have shown that TKR with domeshaped patellae have more superior contact points on the patella and a greater patellar tilt angle in the superiorinferior direction than that with native or mobile-bearing patellae. ${ }^{27,28}$ It may be that the domed patellar design is more susceptible to the effect of a flexion contracture and lateral patellar displacement than the non-resurfaced patella, perhaps because of increased patellofemoral contact stress or shear stress. It has also been hypothesised that increased patellofemoral contact stress in resurfaced TKR may be a consequence of the material properties of a metal/polyethylene interface, which may result in a decreased ability for expansion under load of both surfaces, and thus decreased potential for pressure dissipation. $^{29}$

The statistical analysis for our study was carried out on the number of knees rather than number of patients. This is a potential problem because of a violation of the assumption of independence of observations, and may result in an underestimation of the standard error of the mean. However, in our study, there were no patients with bilateral TKR who contributed both knees to either treatment group. Furthermore, the results were checked by selecting only the knee with the highest level of pain for analysis. The results did not differ substantially or alter the conclusions of the study in terms of the difference in knee pain scale score change $(4.0, \mathrm{p}=0.711,95 \% \mathrm{CI}-6.5$ to 9.5), or the risk of anterior knee pain for TKR without patellar resurfacing (OR 0.51, $\mathrm{p}=0.092,95 \%$ CI 0.24 to 1.12).

The results of our study indicate no superiority of patellar resurfacing over patelloplasty in a TKR system with an anatomical femoral component and a domed patellar component. They contrast strongly with those of our previous study, suggesting that the design of both the femoral and patellar components may be an important consideration in the decision as to whether or not to resurface the patella.

The authors would like to acknowledge Mr K. Kozak and Professor B. Nivbrant as participating surgeons and Ms V. Petrov for her assistance with data collection.

The author or one or more of the authors have received or will receive benefits for personal or professional use from a commercial party related directly or indirectly to the subject of this article. In addition, benefits have been or will be directed to a research fund, foundation, educational institution, or other nonprofit organisation with which one or more of the authors are associated.

\section{References}

1. Burnett RS, Bourne RB. Indications for patellar resurfacing in total knee arthroplasty. J Bone Joint Surg [Am] 2003;85-A:728-45.

2. Bourne RB, Burnett RS. The consequences of not resurfacing the patella. Clin Orthop 2004;428:166-9.

3. Forster MC. Patellar resurfacing in total knee arthroplasty for osteoarthritis: a systematic review. Knee 2004;11:427-30.

4. Holt GE, Dennis DA. The role of patellar resurfacing in total knee arthroplasty. Clin Orthop 2003;416:76-83.

5. Nizard RS, Biau D, Porcher R, et al. A meta-analysis of patellar replacement in total knee arthroplasty. Clin Orthop 2005;432:196-203.

6. Pakos EE, Ntzani EE, Trikalinos TA. Patellar resurfacing in total knee arthroplasty: a meta-analysis. J Bone Joint Surg [Am] 2005;87-A:1438-45.

7. Kulkarni SK, Freeman MA, Poal-Manresa JC, Asencio JI, Rodriguez JJ. The patellofemoral joint in total knee arthroplasty: is the design of the trochlea the critical factor? J Arthroplasty 2000;15:424-9.

8. Barrack RL. Orthopaedic crossfire: all patellae should be resurfaced during primary total knee arthroplasty: in opposition. J Arthroplasty 2003;18:35-8.

9. Matsuda S, Ishinishi T, Whiteside LA. Contact stresses with an unresurfaced patella in total knee arthroplasty: the effect of femoral component design. Orthopedics 2000;23:213-18.

10. Tanzer M, McLean CA, Laxer E, Casey J, Ahmed AM. Effect of femoral component designs on the contact and tracking characteristics of the unresurfaced patella in total knee arthroplasty. Can J Surg 2001;44:127-33.

11. Insall JN, Dorr LD, Scott RD, Scott WN. Rationale of the Knee Society clinical rating system. Clin Orthop 1989;248:13-14.

12. Rejeski WJ, Ettinger WH Jr, Shumaker $\mathbf{S}$, et al. The evaluation of pain in patients with knee osteoarthritis: the knee pain scale. J Rhemato/ 1995;22:1124-9.

13. Lingard EA, Katz JN, Wright RJ, Wright EA, Sledge CB. Validity and responsiveness of the Knee Society Clinical Rating System in comparison with the SF-36 and the WOMAC. J Bone Joint Surg [Am] 2001;83-A:1856-64.

14. Gomes LS, Bechtold JE, Gustilo RB. Patellar prosthesis positioning in total knee arthroplasty: roentgenographic study. Clin Orthop 1988;236:72-81.

15. Portney LG, Watkins MP. Foundations of clinical research: applications to practice. Second ed. New Jersey: Prentice Hall, 2000:593-5.

16. Wood DJ, Smith AJ, Collopy D, et al. Patellar resurfacing in total knee arthroplasty: a prospective, randomised trial. J Bone Joint Surg [Am] 2002;84-A:187-93.

17. Parvizi J, Rapuri VR, Saley KJ, et al. Failure to resurface the patella during total knee arthroplasty may result in more knee pain and secondary surgery. Clin Orthop 2005:438:191-6.

18. Burnett RS, Haydon CM, Rorabeck CH, Bourne RB. Patella resurfacing versus nonresurfacing in total knee arthroplasty: results of a randomized controlled clinical trial at a minimum of 10 years' followup. Clin Orthop 2004;428:12-25.

19. Hsu HP, Walker PS. Wear and deformation of patellar components in total knee arthroplasty. Clin Orthop 1989;246:260-5.

20. Collier JP, McNamara JL, Surprenant VA, Jensen RE, Surprenant HP. All-polyethylene components are not the answer. Clin Orthop 1991;273:198-203.

21. Matsuda S, Ishinishi T, White SE, Whiteside LA. Patellofemoral joint after total knee arthroplasty: effect on contact area and contact stress. J Arthroplasty 1997; $12: 790-7$

22. Singerman R, Gabriel SM, Maheshwer CB, Kennedy JW. Patellar contact forces with and without patellar resurfacing in total knee arthroplasty. J Arthroplasty 1999;14:603-9.

23. Rosenberg AG, Andriacchi TP, Barden R, Galante J0. Patellar component failure in cementless total knee arthroplasty. Clin Orthop 1988;236:106-14.

24. Barrack RL, Bertot AJ, Wolfe MW, et al. Patellar resurfacing in total knee arthroplasty: a prospective, randomized, double-blind study with five to seven years of followup. J Bone Joint Surg [Am] 2001;83-A:1376-81.

25. Waters TS, Bentley G. Patellar resurfacing in total knee arthroplasty: a prospective, randomized study. J Bone Joint Surg [Am]2003;85-A:212-17.

26. Smith AJ, Lloyd DG, Wood DJ. A kinematic and kinetic analysis of walking after total knee arthroplasty with and without patellar resurfacing. Clin Biomech 2006;21:379-86.

27. Stiehl JB, Komistek RD, Dennis DA, Paxson RD. Fluoroscopic analysis of kinematics after posterior-cruciate-retaining knee arthroplasty. J Bone Joint Surg [Br] 1995;77B:884-9

28. Stiehl JB, Komistek RD, Dennis DA, Keblish PA. Kinematics of the patellofemoral joint in total knee arthroplasty. J Arthroplasty 2001;16:706-14.

29. Lee T0, Gerken AP, Glaser FE, Kim WC, Anzel SH. Patellofemoral joint kinematics and contact pressures in total knee arthroplasty. Clin Orthop 1997;340:257-66. 